\title{
Morphology evolution and local electric properties of Au nanoparticles on $\mathrm{ZnO}$ thin films
}

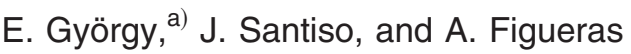 \\ Consejo Superior de Investigaciones Cientificas, Instituto de Ciencia de Materiales de Barcelona, \\ Campus UAB, 08193 Bellaterra, Spain
}

\begin{abstract}
A. Giannoudakos ${ }^{\text {b) }}$ and M. Kompitsas
National Hellenic Research Foundation, Theoretical and Physical Chemistry Institute, Vasileos Konstantinou Avenue 48, 11635 Athens, Greece
\end{abstract}

\author{
I. N. Mihailescu \\ Institute of Atomic Physics, P.O. Box MG 54, Bucharest V, Romania
}

(Received 28 February 2005; accepted 6 September 2005; published online 18 October 2005)

\begin{abstract}
We report the sequential two-step pulsed laser deposition of nanostructures consisting of $\mathrm{ZnO}$ thin films covered with $\mathrm{Au}$ nanoparticles. $\mathrm{Zn}$ and $\mathrm{Au}$ targets were alternatively submitted to pulses generated by a frequency tripled $\mathrm{Nd}$ :yttrium aluminium garnet $(\lambda=355 \mathrm{~nm}, \tau \sim 10 \mathrm{~ns}, \nu=10 \mathrm{~Hz})$ laser. The $\mathrm{ZnO}$ films were synthesized in $20 \mathrm{~Pa}$ oxygen pressure, whereas the subsequent $\mathrm{Au}$ coverage was applied in vacuum. The dynamics of $\mathrm{Au}$ nanoparticles grown on the $\mathrm{ZnO}$ thin-films surface was studied by atomic force microscopy as a function of number of laser pulses applied for the ablation of the Au target. The nanoparticle diameters increase with the number of laser pulses while their height decreases. The local electric properties of the uncoated (reference) and coated with $\mathrm{Au} \mathrm{ZnO}$ thin films was investigated by current sensing mode atomic force microscopy. (C) 2005 American Institute of Physics. [DOI: 10.1063/1.2089166]
\end{abstract}

\section{INTRODUCTION}

Controlled growth of nanostructures that consist of small noble-metal particles with a few up to a few tens of nanometer dimensions on the surface of wide-band-gap transition metal oxide surfaces focused considerable attention during the last years. ${ }^{1-4}$ Indeed, due to their valuable electronic and optical properties, the noble-metal/transition-metal oxide composite systems find application in numerous technological areas, such as oxide supported metal catalysts and gas sensors. ${ }^{1,2}$ Moreover, the experiments show that in biosensors the gold nanoparticles enhance enzyme adhesion. ${ }^{5,6}$ It was found that these special, nanoparticle-supported gas or biosensors exhibit high sensitivity, good reproducibility, and long-term stability. ${ }^{1}$

As it is known, the size and the shape of metal particles on oxide surfaces determine the reactivity of the noblemetal/transition-metal oxide systems to the ambient gas atoms or molecules. ${ }^{1,4}$ The various growth modes of metal particles on thin oxide films have been reviewed in Ref. 1 for different noble-metal/metal oxide combinations. The growth of noble metal on a given transition-metal oxide surface was shown to be dependent on the nature of the deposited noble metals. In the particular case of $\mathrm{ZnO}$ surface, layer-by-layer growth is characteristic for Ni (Ref. 7) and Pt. ${ }^{8}$ Conversely, $\mathrm{Cu}$ on $\mathrm{ZnO}$ displays a two-dimensional (2D) island growth, ${ }^{1}$ or Stranski-Krastanov nucleation, ${ }^{9}$ whereas Pd shows a layer-by-layer, ${ }^{7}$ or Volmer-Weber three-dimensional (3D) island clusters formation. ${ }^{10}$

\footnotetext{
a) Electronic mail: egyorgy@icmab.es

b) Also at National Technical University of Athens, Department of Chemical Engineering, 15780 Zografou, Athens, Greece.
}

The purpose of our investigations is the study of the evolution of the shape and size of Au particles on the surface of $\mathrm{ZnO}$ thin films in view of the determination of the growth mode, as a function of increasing Au coverage. Both $\mathrm{ZnO}$ and $\mathrm{Au}$ were grown by pulsed laser deposition. This work is a development of investigations of $\mathrm{ZnO}$ thin films deposition on $\mathrm{Si}(001)$ and $\mathrm{SiO}_{2}$ (001) substrates. ${ }^{11,12}$ These previous studies permitted us to identify the optimum experimental conditions that allow for the growth of $\mathrm{ZnO}$ films with c-axis-oriented crystal structure.

To date only a few groups reported the growth of $\mathrm{Au}$ nanoparticles on $\mathrm{ZnO}$ films surfaces. ${ }^{13-15}$ In the mentioned studies the $\mathrm{Au} / \mathrm{ZnO}$ nanostructures were grown via evaporation ${ }^{13}$ or rf sputtering methods. ${ }^{14,15}$ This work presents the extension of pulsed laser deposition technique to the study of the evolution of Au nanoparticles growth on $\mathrm{ZnO}$ thin films for sensing applications.

\section{EXPERIMENTAL DETAILS}

The deposition of the Au nanostructures was carried out inside a stainless-steel vacuum chamber. Prior to each laser irradiation the chamber was evacuated down to a residual pressure of $6 \times 10^{-4} \mathrm{~Pa}$. To promote the ablation of the $\mathrm{Zn}$ and Au targets the pulses of a Quantel Mo. YG851 Nd:yttrium aluminium garnet (YAG; $\lambda=355 \mathrm{~nm}, \tau_{\mathrm{FWHM}} \sim 10 \mathrm{~ns}$ ) laser were applied at a repetition rate of $10 \mathrm{~Hz}$. The laser fluence was estimated at $6.6 \mathrm{~J} / \mathrm{cm}^{2}$.

To avoid drilling under the action of multipulse laser irradiation, both the $\mathrm{Zn}$ and $\mathrm{Au}$ targets were placed on a moveable vacuum-compatible computer-controlled XY table. The $\mathrm{Si}$ (001) substrates were positioned parallel to the targets 
at $40 \mathrm{~mm}$ distance. During the growth of the $\mathrm{ZnO} / \mathrm{Au}$ structures, the substrates were heated at a temperature of $300{ }^{\circ} \mathrm{C}$.

The targets and substrates were carefully cleaned with acetone in ultrasonic bath before introducing them into the vacuum chamber. Before the depositions took place the targets were exposed to a preliminary ablation step. During the laser preconditioning a shutter was interposed halfway between the targets and the substrate, parallel to them. According to previous experience this is essential for removing the last contaminants and impurities present on the surface of the targets.

All $\mathrm{ZnO}$ thin films were deposited in $20 \mathrm{~Pa}$ oxygen pressure, under the action of 70000 laser pulses. The subsequent $\mathrm{Au}$ coverage was performed at a $6 \times 10^{-4} \mathrm{~Pa}$ background pressure. Its thickness was varied by increasing the number of laser pulses from 300 to 9000 .

The deposited $\mathrm{ZnO}$ thin films and $\mathrm{Au} / \mathrm{ZnO}$ nanostructures were investigated by atomic force microscopy (AFM) with a PicoSPM apparatus from Molecular Imaging. The films surface morphology was studied by acoustic (dynamic) mode and their local electric properties by current sensing contact mode AFM. ${ }^{16,17}$

\section{RESULTS AND DISCUSSION}

Figure 1 contains the AFM images, surface profiles, and histograms of the grains' (nanoparticles) diameters of the uncovered $\mathrm{ZnO}$ thin films and $\mathrm{Au} / \mathrm{ZnO}$ nanostructures obtained with different number of laser pulses applied for the irradiation of the Au targets. We note that all the histograms of grains' diameters [Figs. 1(c), 1(f), 1(i), and 1(m)] as well as the maximum and average heights were evaluated on $25 \mu \mathrm{m}^{2}$ AFM scan areas.

As can be observed, the surface of the uncovered $\mathrm{ZnO}$ thin film [Figs. 1(a)-1(c)] is composed by grains with maximum diameters of about $50 \mathrm{~nm}$. The maximum height was found to be about $14 \mathrm{~nm}$. The average diameter of the nanoparticles was calculated to be about $20 \mathrm{~nm}$ and the average height around $8 \mathrm{~nm}$.

According to literature, ${ }^{18-21}$ the characteristic elements of $\mathrm{ZnO}$ film surfaces are grains with dimensions from a few tens up to $100-200 \mathrm{~nm}$. The growth and final surface morphology practically do not depend on the films' deposition technique. ${ }^{18-21}$ More precisely, $\mathrm{ZnO}$ film growth is dominated by a 3D tip-type pattern of islands, whenever the film thickness exceeds a characteristic threshold value. ${ }^{18}$ Below this threshold, i.e., at thickness values of a few nanometers, the $\mathrm{ZnO}$ films grow in a layer-by-layer mode and its surface is atomically flat.

The factors which stay at the origin of the 3D growth are not yet completely elucidated. Nevertheless, it was suggested in Ref. 18 that above the critical thickness, misfit dislocations are produced in the growing films structure. Then, the strained epilayer will minimize its energy by $3 \mathrm{D}$ island formation. We mention that the thickness of our $\mathrm{ZnO}$ films measured by wavelength dispersive $\mathrm{x}$-ray spectroscopy was about $80 \mathrm{~nm}$, well above the critical thickness value.

To promote the metal/transition-metal oxide heterostructures growth we covered in a second step with Au the ob-
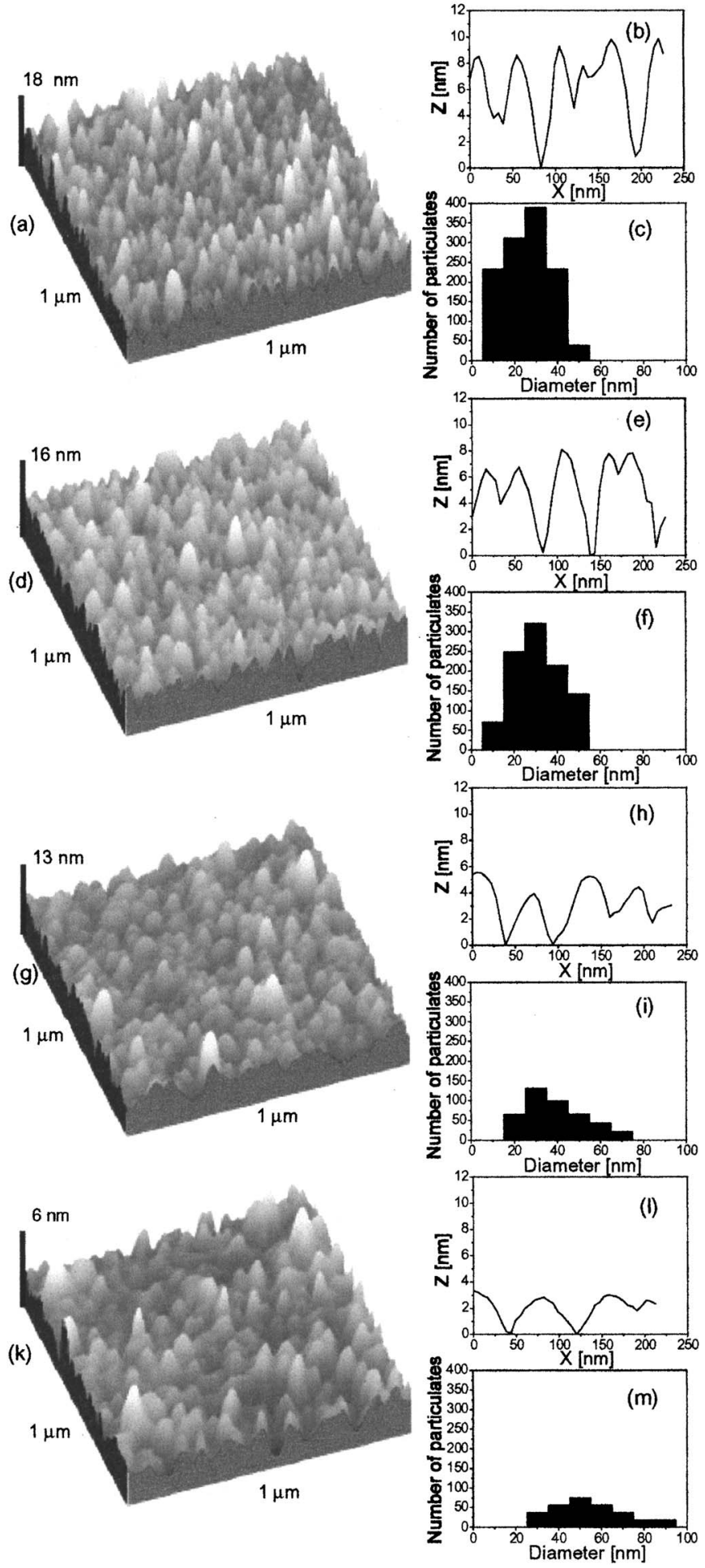

FIG. 1. AFM images, surface profiles, and histograms of nanoparticles diameter counted on $25 \mu \mathrm{m}^{2}$ surface areas for reference $\mathrm{ZnO}$ thin films [(a), (b), and (c)], as well as $\mathrm{ZnO}$ films with $\mathrm{Au}$ coverage obtained with 1200 $[(\mathrm{d}),(\mathrm{e})$, and (f)], $4600[(\mathrm{~g}),(\mathrm{h})$, and (i)], and $9000[(\mathrm{k}),(\mathrm{l})$, and (m)] laser pulses.

tained $\mathrm{ZnO}$ films. To this purpose, the Au targets were submitted to different number of laser. Under visual inspection the uncovered $\mathrm{ZnO}$ films were transparent. On the other hand, the bluelike color of the $\mathrm{ZnO}$ thin films covered with $\mathrm{Au}$ was a first indication of the formation of Au clusters, rather than a continuous yellow metallic Au layer. ${ }^{22}$ 


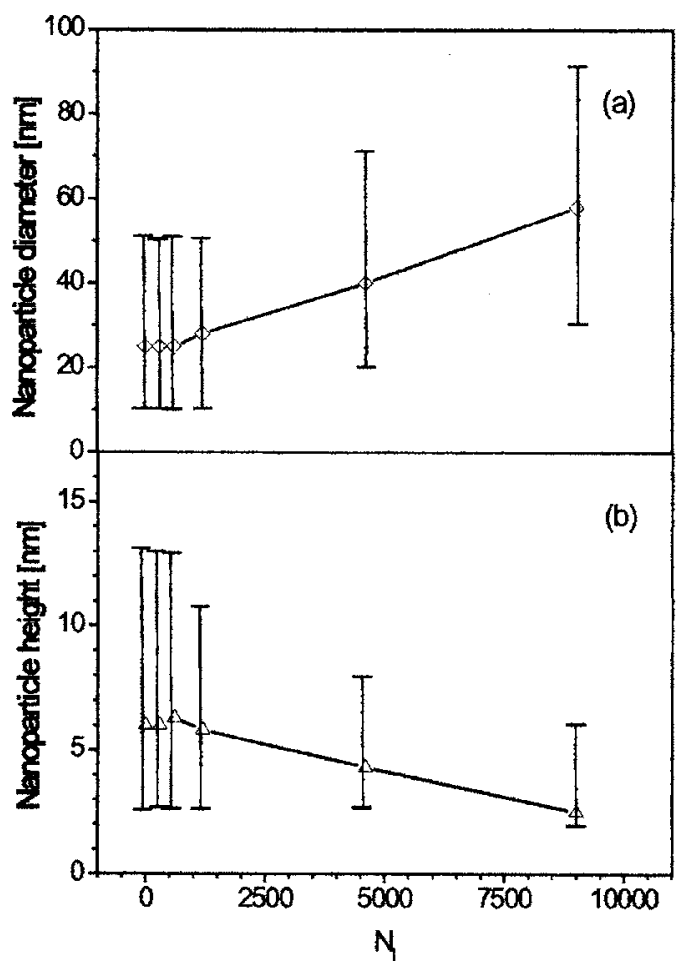

FIG. 2. Nanoparticles (a) average diameter and (b) average height as a function of number of laser pulses applied for the irradiation of the Au targets.

By AFM investigations no significant differences were observed in the surface morphology, grain shape, and dimensions, for laser pulses less than about 1200 [Figs. 1(d)-1(f)], as compared to the uncovered $\mathrm{ZnO}$ films. We note that $\mathrm{Au}$ additive on $\mathrm{TiO}_{2}$ surface has been reported to form agglomerated clusters with comparable shape and size to the semiconductor grains. ${ }^{23}$ Therefore, it should be reasonable to attribute to this effect the similar surface morphologies of the uncovered $\mathrm{ZnO}$ and $\mathrm{Au}$ covered $\mathrm{ZnO}$ thin films.

When continuing the deposition [Figs. $1(\mathrm{~g})-1(\mathrm{~m})]$, the average diameter of the grains increases gradually from about $20 \mathrm{~nm}$ corresponding to the samples obtained with less than 1200 pulses [Fig. 1(f)], to about $50 \mathrm{~nm}$ for the largest number (9000) of laser pulses used for the Au coverage application [Fig. 1(m)]. Conversely, the grains' average height decreases with the increase of the number of laser pulses from about $6 \mathrm{~nm}$ corresponding to the sample obtained with 1200 laser pulses [Fig. 1(e)] to about $2 \mathrm{~nm}$ for the highest number of pulses applied for the ablation of the Au target [Fig. 1(1)]. Consequently, the minimum root-mean-square $(\mathrm{rms})$ roughness of the surfaces decreases from about $4 \mathrm{~nm}$ for the uncovered $\mathrm{ZnO}$ films to about $1 \mathrm{~nm}$ for the $\mathrm{ZnO}$ films with Au coverage obtained with 9000 laser pulses.

On the other hand, the grains' density decreases with the increase of the number of laser pulses applied for the $\mathrm{Au}$ coverage [see Figs. 1(c), 1(f), 1(i), and 1(m)]. This proves the partial coalescence of the Au nanoparticles during their growth. The coalescence of the nanoparticles can also explain the mentioned decrease of their relative height. We note, however, that the transition from spherical to an elliptical morphology with the increase of the metal coverage was reported also in Ref. 24 in the case of Pd clusters. Very small
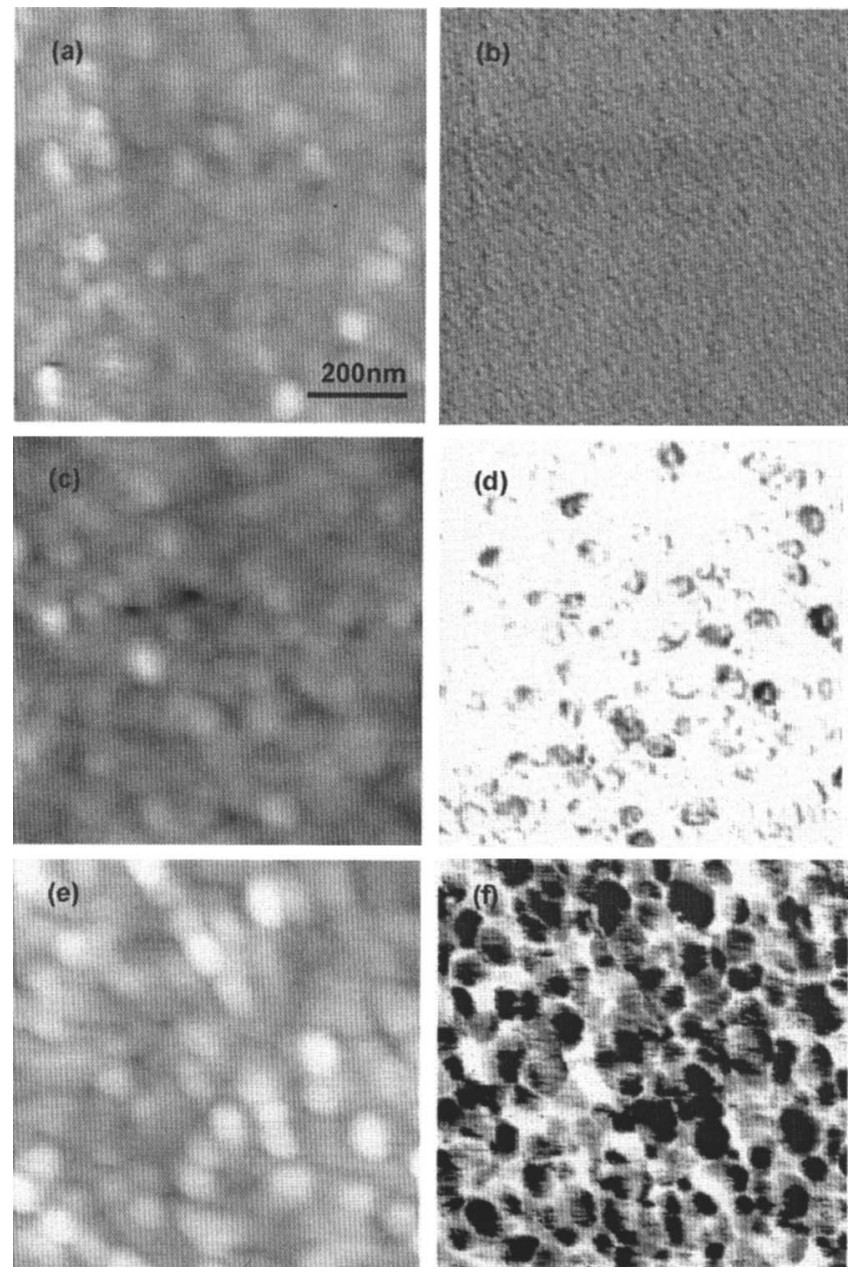

FIG. 3. AFM images and conductivity maps of the same surface areas for reference $\mathrm{ZnO}$ film [(a) and (b)] and for $\mathrm{ZnO}$ films with Au coverage obtained with 1200 [(c) and (d)] as well as 9000 [(e) and (f)] laser pulses. The magnification is the same for all images and maps.

clusters $(<4 \mathrm{~nm})$ were found to mostly exhibit a spherical shape, while for larger particles the lateral size exceeds the cluster height.

Figure 2 summarizes the results concerning the evolution of the grains' average diameters and heights, respectively, as a function of applied laser pulses to obtain the $\mathrm{Au}$ coverage. One clearly notices that the increase of the number of laser pulses results in the increase of the nanoparticles diameter, accompanied by the decrease of their height.

The 3D type growth mode of metal particles on oxide surfaces was associated to the metal affinity to oxygen. The more reactive is the metal, the more bidimensional is the islands' growth. ${ }^{25}$ Thus, the observed 3D growth of $\mathrm{Au}$ on the $\mathrm{ZnO}$ thin films surfaces could be due to the reduced reactivity of Au to oxygen. In other studies the existence of a critical coverage at which the growth mode switches from two-to three-dimensional islands was reported for $\mathrm{Cu}$ on $\mathrm{ZnO}{ }^{26}$ and for $\mathrm{Au}$ on $\mathrm{TiO}_{2}$ surface. ${ }^{27}$ The transition from two- to a three-dimensional growth mode was explained by the metal adatom migration and island nucleation.

The local electric properties of the Au clusters were further studied using current sensing mode atomic force microscopy. Figure 3 shows the typical AFM images and the cor- 


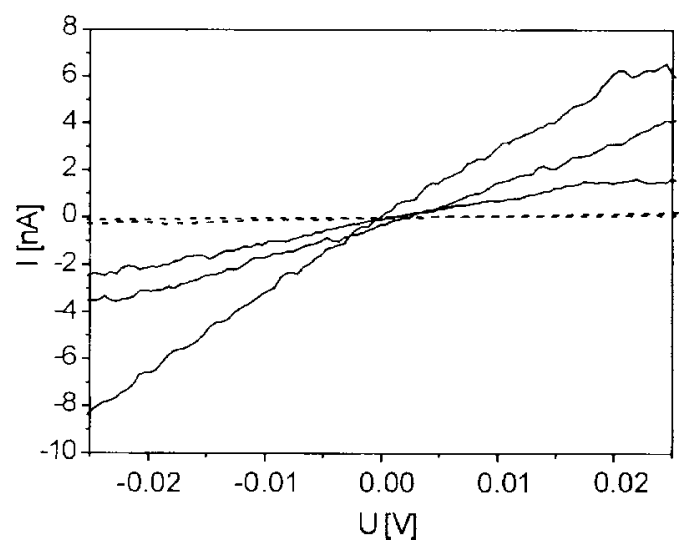

FIG. 4. Local $I-V$ characteristics obtained in the center (continuous lines) and at the border (dashed lines) of the nanoparticles for a $\mathrm{ZnO}$ film with $\mathrm{Au}$ coverage obtained with 9000 laser pulses.

responding conductivity maps of $1 \times 1 \mu \mathrm{m}^{2}$ area of uncovered $\mathrm{ZnO}$ surface (a) and (b), as well as of $\mathrm{ZnO}$ films with Au coverage which were obtained with 1200 [(c) and (d)] and 9000 laser pulses [(e) and (f)]. The conductivity map of uncovered $\mathrm{ZnO}$ surface is uniform, whereas the contrast of the $\mathrm{Au}$ covered surfaces proves the existence of differently conducting zones.

In order to find out more about the local conductivity on the $\mathrm{Au}$ covered surface, we recorded the current-voltage $(I-V)$ curves in different zones. Typical curves, measured in the center of the dark areas corresponding to the Au nanoparticles (continuous lines), and close to their borders measured on the light zones (dashed lines) are given in Fig. 4. As can be observed, the center of the nanoparticles have a metallic character, while the zones close to their borders exhibit insulating behavior with a zero current value. Similar studies of local electric properties have been performed in the case of $\mathrm{Au}$ clusters deposited on $\mathrm{MgO}$ and $\mathrm{TiO}_{2}$ surfaces. ${ }^{28}$ The authors found distinct $I-V$ curves depending on the cluster size. The gradual transition from insulator up to the bulk metallic electronic structure proceeds along with the increase of the metal cluster sizes. The metallic behavior becomes obvious for clusters with diameters larger than about $5 \mathrm{~nm}$. These data are in good agreement with our results where the deposited Au clusters have diameters of the order of a few tens of nanometer. We recall that in sensor applications the presence of metallic clusters is required to ensure better selectivity and sensitivity, as well as the diminishment of the response time and working temperature as compared to the base, uncovered transition-metal oxide. ${ }^{1-6,23}$

\section{CONCLUSION}

We studied the evolution of the Au nanoparticles morphology deposited on the surface of $\mathrm{ZnO}$ thin films. Both the $\mathrm{Au}$ coverage and the underlying $\mathrm{ZnO}$ films were grown by pulsed laser deposition. A frequency tripled $\mathrm{Nd}$ :YAG laser $\left(\lambda=355 \mathrm{~nm}, \quad \tau_{\mathrm{FWHM}} \sim 10 \mathrm{~ns}, \quad \nu=10 \mathrm{~Hz}\right)$ was used for the multipulse irradiation of the $\mathrm{Zn}$ and $\mathrm{Au}$ targets, respectively.
The deposition of the $\mathrm{ZnO}$ films was performed in a lowpressure oxygen atmosphere, while the Au coverage was deposited in vacuum. The AFM studies showed that the nanoparticles diameter increases with the increase of the amount of deposited $\mathrm{Au}$, while their height decreases. The investigations of the local electric properties of the Au covered $\mathrm{ZnO}$ surfaces revealed the metallic character of the Au nanoparticles grown on the insulating $\mathrm{ZnO}$ thin films surface. These features justify the use of the obtained heterostructures in gas sensor applications since metallic nanoparticles act as preferential catalytic adsorption sites in reducing chemical environment.

\section{ACKNOWLEDGMENTS}

Financial support from NATO (PST.CLG 980464) and Ministry of Education and Science of the Spanish Government (MAT2002-03075) is acknowledged with thanks.

${ }^{1}$ C. T. Campbell, Surf. Sci. Rep. 27, 111 (1997).

${ }^{2}$ N. Yamazoe, Sens. Actuators B 5, 7 (1991).

${ }^{3}$ D. A. Chen, M. C. Bartelt, R. Q. Hwang, and K. F. McCarty, Surf. Sci. 450, 78 (2000).

${ }^{4}$ H. J. Freund, Surf. Sci. 500, 271 (2002).

${ }^{5}$ I. Bontidean, J. R. Lloyd, J. L. Hobman, J. R. Wilson, E. Csoregi, B. Mattiasson, and N. L. Brown, J. Inorg. Biochem. 79, 225 (2000).

${ }^{6}$ J. Jia, B. Wang, A. Wu, G. Cheng, Z. Li, and S. Dong, Anal. Chem. 74, 2217 (2002).

${ }^{7}$ D. Schmeisser and K. Jacobi, Surf. Sci. 88, 138 (1979).

${ }^{8}$ W. T. Petrie and J. M. Vohs, J. Chem. Phys. 101, 8098 (1994).

${ }^{9}$ S. V. Didziulis, K. D. Butcher, S. L. Cohen, and E. I. Solomon, J. Am. Chem. Soc. 111, 7110 (1989).

${ }^{10}$ H. Jacobs, W. Mokwa, D. Kohl, and G. Heiland, Surf. Sci. 160, 217 (1985).

${ }^{11}$ E. György, J. Santiso, A. Giannoudakos, M. Kompitsas, I. N. Mihailescu, and D. Pantelica, Appl. Surf. Sci. 248(1-4), 147 (2005).

${ }^{12}$ E. György, J. Santiso, G. Socol, I. N. Mihailescu, C. Ducu, and S. Ciuca, Proc. SPIE 5830, 50 (2005).

${ }^{13}$ E. F. Wasserman and K. Polacek, Surf. Sci. 28, 77 (1971).

${ }^{14}$ M. Ay, A. Nefedov, and H. Zabel, Appl. Surf. Sci. 205, 329 (2003).

${ }^{15}$ U. Pal, J. Garcia-Serrano, G. Casarrubias-Segura, N. Koshizaki, T. Sasaki, and S. Terahuchi, Sol. Energy Mater. Sol. Cells 81, 339 (2004).

${ }^{16}$ O. Schneegans, F. Houzé, R. Meyer, and L. Boyer, IEEE Trans. Compon., Packag. Manuf. Technol., Part A 21, 76 (1998).

${ }^{17}$ Y. Iseri, M. Honda, Y.-D. Kim, T. Ando, W. Choi, and H. Tomokage, J. Phys.: Condens. Matter 16, S171 (2004).

${ }^{18}$ T. B. Hur, Y. H. Hwang, H. K. Kim, and H. L. Park, J. Appl. Phys. 96, 1740 (2004).

${ }^{19}$ E. M. Kaidashev et al., Appl. Phys. Lett. 82, 3901 (2003).

${ }^{20}$ Y. G. Wang, S. P. Lau, H. W. Lee, S. F. Yu, B. K. Ray, X. H. Zhang, K. Y. Tse, and H. H. Hng, J. Appl. Phys. 94, 1597 (2003).

${ }^{21}$ Y. Sun, J. B. Ketterson, and G. K. L. Wong, Appl. Phys. Lett. 77, 2322 (2000).

${ }^{22}$ D. Barreca, A. Gasparotto, E. Tondello, G. Bruno, and M. Losurdo, J. Appl. Phys. 96, 1655 (2004).

${ }^{23}$ A. Cabot, J. Arbiol, J. R. Morante, U. Weimar, N. Barsan, and W. Gopel, Sens. Actuators B 70, 87 (2000).

${ }^{24}$ J. Schneider, C. Wambach, B. Pennemann, and K. Wandelt, Langmuir 15, 5765 (1999).

${ }^{25}$ U. Diebold, J. M. Pan, and T. E. Madey, Phys. Rev. B 47, 3868 (1993).

${ }^{26}$ J. Yoshihara, S. C. Parker, and C. T. Campbell, Surf. Sci. 439, 153 (1999).

${ }^{27}$ S. C. Parker, A. W. Grant, V. A. Bondzie, and C. T. Campbell, Surf. Sci. 441, 10 (1999).

${ }^{28}$ C. Xu, W. S. Oh, G. Liu, D. Y. Kim, and D. W. Goodman, J. Vac. Sci. Technol. A 15, 1261 (1997). 